\title{
Prevalência de hemorragia peri-intraventricular em recém-nascidos de muito baixo peso e/ou com idade gestacional menor que 34 semanas e relação com o uso antenatal de corticoide e de sulfato de magnésio em maternidade terciária.
}

\section{Prevalence of peri-intraventricular hemorrhage in newborn very low birth weight and/or less than 34 weeks of age gestational and relation with antenatal use of corticosteroid and magnesium sulfate ata tertiary maternity}

\begin{abstract}
Samila Andrade Vasconcelos ${ }^{1}$. Maria Francielze Holanda Lavor ${ }^{2}$. Daniela Monteiro Ferreira ${ }^{3}$.
1 Médica pediatra, concludente da Residência Médica em Neonatologia da Maternidade Escola Assis Chateaubriand (MEAC), Fortaleza, Ceará, Brasil. 2 Médica pediatra e neonatologista, Mestra em Saúde Pública pela Universidade Federal do Ceará (UFC), médica assistente da Unidade Neonatal da Maternidade Escola Assis Chateaubriand (MEAC-UFC), Fortaleza, Ceará, Brasil. 3 Médica pediatra e neonatologista, Mestranda em Saúde da Criança e do Adolescente pela Universidade Estadual do Ceará (UECE), médica assistente da Unidade Neonatal da Maternidade Escola Assis Chateaubriand (MEAC-UFC), Fortaleza, Ceará, Brasil.
\end{abstract}

\section{RESUMO}

Objetivos: conhecer a prevalência de hemorragia peri-intraventricular (HPIV) em recém-nascidos de muito baixo peso (RNMBP) e/ou menor que 34 semanas de idade gestacional (IG). Verificar relação da patologia com o uso antenatal de corticoide e de sulfato de magnésio. Metodologia: estudo retrospectivo e transversal realizado por meio da revisão de prontuários do serviço de maternidade terciária no período de $1^{\circ}$ de julho a 31 de dezembro de 2015. Resultados/Discussão: de 110 pacientes incluídos, todos tinham IG menor que 34 semanas e 41 tiveram HPIV (37,3\%) e houve predomínio da hemorragia grau 1 de Papile $(63,4 \%)$. Houve preponderância de HPIV na faixa de peso ao nascer de 1000-1499g (48,8\%) e na faixa de IG de 28 semanas a 31 semanas e 6 dias $(53,7 \%)$. A taxa de exposição ao corticoide na amostra total incluída foi de 77,3\%, semelhante à exposição no grupo com HPIV (75,6\%). A exposição ao sulfato de magnésio foi de 41,8\% na amostra total e 34,1\% no grupo com HPIV. Conclusão: a relação entre HPIV e prematuros com IG menor que 34 semanas corresponde a 37,3\%. A grande maioria dos casos de HPIV encontrados foram em recém-nascido (RN) com peso de nascimento menor que $1500 \mathrm{~g}(85,4 \%)$.

Palavras-chave: Hemorragia intracraniana. Recém-nascido. Corticoide. Sulfato de magnésio.

\section{ABSTRACT}

Objectives: this study aims to determine the prevalence of peri-intraventricular hemorrhage (PIVH) in newborns very low birth weight (NVLBW) and/or less than 34 weeks of gestational age (GA). Also to check the relation or not between PIVH and the antenatal use of corticosteroids and magnesium sulfate. Methods: a retrospective and cross-sectional study carried out by reviewing medical records of tertiary maternity service for the period from $1^{\text {st }}$ July to 31 December 2015. Results/Discussion: all 110 patients included had GA less than 34 weeks and 41 (37.3\%) were diagnosed with PIVH and there was a predominance of grade 1 hemorrhage Papile (63.4\%). There was a preponderance of PIVH in the birth weight range of $1000-1499 \mathrm{~g}(48.8 \%)$ and GA range 28 weeks to 31 weeks and 6 days $(53.7 \%)$. Corticosteroid exposure rate in the included sample was $77.3 \%$, similar to exposure in the group with PIVH (75.6\%). Magnesium sulfate exposure rate was $41.8 \%$ in the total sample and $34.1 \%$ in the group with PIVH. Conclusion: the relation between PIVH and preterm infants with GA less than 34 weeks corresponds to $37.3 \%$. The vast majority of cases of PIVH found were in infants with birth weight less than $1500 \mathrm{~g}(85.4 \%)$.

Keywords: Intracranial hemorrhages. Infant, newborn. Corticosteroids. Magnesium sulfate.

Autor correspondente: Samila Andrade Vasconcelos, Rua Coronel Nunes de Melo, s/n, Rodolfo Teófilo, Fortaleza, Ceará. CEP: $60430-270$. Telefone: +55 85 3366-8528. E-mail: samila_av@yahoo.com.br

Conflito de interesses: Não há qualquer conflito de interesses por parte de qualquer um dos autores.

Recebido em: 17 Mai 2016; Revisado em: 27 Jun 2016; Aceito em: 27 Jul 2016. 


\section{INTRODUÇĀO}

Embora tenham crescido os avanços na área da perinatologia e melhorado significativamente o prognóstico dos recém-nascidos e reduzida a mortalidade neonatal, observa-se notória e crescente morbidade neurológica em neonatos muito prematuros e de menor peso ao nascer. ${ }^{1}$

Entre os recém-nascidos prematuros (RNPT) extremos, pesando entre 500-750g, as hemorragias cerebrais peri-intraventriculares (HPIV) ocorrem em aproximadamente $45 \%$ desses pacientes. ${ }^{2}$ Cerca de $50-75 \%$ dos prematuros com HPIV desenvolveram paralisia cerebral, retardo mental e/ ou hidrocefalia. Aproximadamente $25 \%$ dos não-deficientes desenvolveram distúrbios psiquiátricos. ${ }^{3}$

As lesões cerebrais oriundas de eventos de isquemia, hipóxia e das HPIV, esta quase exclusiva em pré-termos, contribuem significativamente para o aumento da morbimortalidade nos prematuros. Essas hemorragias, tanto intraparenquimatosas como na matriz germinativa, têm como etiologia a participação de vários fatores, entre eles: maior vulnerabilidade dos vasos cerebrais dos prematuros, oscilações do fluxo sanguíneo cerebral e alterações da pressão intracraniana. É pouco comum o surgimento dessa patologia em crianças a termo, cujos mecanismos etiológicos não são muito bem caracterizados nesses pacientes. ${ }^{4,5}$

A região da matriz germinativa é bastante suscetível à hemorragia devido a maior vulnerabilidade de seus vasos, a qual é justificada por sua simples constituição por endotélio apenas. Essa frágil vasculatura é sujeita às variações de fluxo sanguineo cerebral, que, nos bebês a termo, possui seus próprios mecanismos de auto-regulação que tentam mantê-lo constante diante de variações na pressão arterial sistêmica. Nos prematuros, esses mecanismos estão ausentes, o que implica em maior propensão às oscilações de pressão arterial e, consequentemente, maior exposição dos vasos cerebrais a danos e lesões e à ocorrência de HPIV. ${ }^{6}$

Pode-se classificar a HPIV, segundo Papile, em 4 graus, de acordo com os achados da ultrassonografia transfontanelar, conforme o grau de distribuição da hemorragia: Grau Ihemorragia restrita à matriz germinativa; Grau II - hemorragia intraventricular sem dilatação dos ventrículos; Grau III hemorragia intraventricular com dilatação dos ventrículos; e Grau IV - hemorragia intraparenquimatosa. ${ }^{7}$

A tentativa de minimizar o risco para HPIV, já começa no período antenatal, através da redução do trabalho de parto prematuro, uso de tocolíticos, uso antenatal de corticoide e de sulfato de magnésio, além de vigilância e tratamento de infecções maternas e/ou amniorrexe prematura.

Entre os objetivos deste estudo estão: conhecer a prevalência de HPIV nos RNMBP (ou seja, com peso de nascimento menor que $1500 \mathrm{~g}$ ) e/ou com IG menor que 34 semanas no serviço de neonatologia da Maternidade Escola Assis Chateaubriand (MEAC), estratificar os dados encontrados conforme a classificação de Papile, faixas de peso e de idade gestacional, além de relacionar a prevalência de HPIV com o uso antenatal de corticoide e de sulfato de magnesio.

\section{METODOLOGIA}

Trata-se de um estudo retrospectivo e transversal, que se realizou por meio da revisão de prontuários do Serviço de Arquivo Médico da MEAC, maternidade pública terciária de Fortaleza, por meio de questionário simples. A população do estudo foi constituída por crianças nascidas nesse serviço entre $1^{\circ}$ de julho a 31 de dezembro de 2015 , com peso de nascimento menor que 1500 gramas e/ou com idade gestacional menor que 34 semanas. Foram incluídos apenas os bebês que realizaram ultrassonografia (US) transfontanelar para diagnóstico de HPIV, em qualquer idade, e, a partir de então, analisou-se a prevalência de HPIV e, em seguida, relação com o uso antenatal de sulfato de magnésio e corticoide (uma ou duas doses de dexametasona, recomendada pelo protocolo do serviço).

Foram excluídos do estudo os recém-nascidos com malformação congênita e os que tiveram Apgar menor que sete no quinto minuto de vida.

A prevalência de HPIV foi estratificada em graus, de acordo com a classificação de Papile, e analisada também de acordo com as seguintes variáveis: peso de nascimento, sexo, idade gestacional e tipo de parto. Foi considerado o peso de nascimento aferido na sala de parto e os pacientes foram agrupados em faixas de peso $(<500 \mathrm{~g}, 500-999 \mathrm{~g}, 1000-$ $1499 \mathrm{~g}$ e $>1499 \mathrm{~g}$ ), para comparação das taxas de prevalência de HPIV. Considerou-se a idade gestacional determinada pela ecografia obstétrica precoce e, quando ausente, pelo cálculo a partir do primeiro dia da data da última menstruação (DUM). $\mathrm{Na}$ ausência dos dois métodos anteriores, a IG foi estimada pelo exame físico do recém-nascido.

O diagnóstico foi feito utilizando aparelho ultrassonográfico Xario $X G$ e um transdutor de $5 \mathrm{mHz}$. Os exames foram realizados na beira do leito com os pacientes dentro da incubadora, segundo o protocolo do nosso serviço, que tem por base avaliar todos os recém-nascidos com peso inferior a $1500 \mathrm{~g}$ ou com idade gestacional de nascimento menor que 34 semanas, seriadamente, após 72 horas de vida (segundo esse protocolo, o primeiro exame é realizado entre o $4^{\circ} \mathrm{e}$ o $7^{\circ}$ dia de vida e repetido semanalmente caso detectada HPIV).

O projeto foi aprovado pelo Comitê de Ética em Pesquisa da Maternidade Escola Assis Chateaubriand-Universidade Federal do Ceará com o número de parecer 1.471.767. Foram respeitados todos os princípios éticos para pesquisa com seres humanos, segundo as normas do Conselho Nacional de Saúde e está de acordo com os critérios estabelecidos pela Declaração de Helsinki com as suas modificações.

Todos os resultados obtidos foram analisados no programa EpiInfo. 


\section{RESULTADOS}

No período analisado, entre $1^{\circ}$ de julho a 31 de dezembro de 2015, nasceram 154 RNMBP e/ou com IG menor que 34 semanas. Destes, foram 110 recém-nascidos incluídos no nosso estudo e os outros 44 excluídos, ou por serem asfixiados (Apgar menor que 7 no $5^{\circ}$ minuto de vida), ou por malformação congênita, ou por falta do exame diagnóstico.

Dos 110 pacientes incluídos, todos tinham IG menor que 34 semanas e 41 deles tiveram diagnóstico de HPIV (37,3\%) e houve predomínio da hemorragia grau 1 de Papile (63,4\%), logo seguida pela hemorragia grau $2(29,3 \%)$. Houve apenas 1 caso $(2,4 \%)$ de HPIV III e 2 casos $(4,9 \%)$ de HPIV IV. (Tabela 1$)$

Tabela 1. Prevalência de HPIV de acordo com a classificação de Papile.

\begin{tabular}{ll}
\hline HPIV $(\mathrm{n}=41)$ & \\
\hline Grau I & $26(63,4 \%)$ \\
Grau II & $12(29,3 \%)$ \\
Grau III & $1(2,4 \%)$ \\
Grau IV & $2(4,9 \%)$ \\
\hline
\end{tabular}

Além disso, na análise dos dados, pôde-se estratificar a prevalência de HPIV de acordo com faixas de peso ao nascer e de idade gestacional (Tabelas 2 e 3). O menor peso ao nascer encontrado na amostra incluída foi de $635 \mathrm{~g}$ e o maior de $2615 \mathrm{~g}$. Houve preponderância de HPIV na faixa de peso de 1000-1499g (20 casos, 48,8\%). 6 neonatos (14,6\%) diagnosticados com HPIV pesaram mais que $1500 \mathrm{~g}$ e foram incluídos no estudo devido à idade gestacional menor que 34 semanas. Os 2 casos de HPIV grau IV pesaram entre $500-999 \mathrm{~g}$ e tinham IG menor que 28 semanas. Houve maior prevalência de hemorragia na faixa de 28 semanas a 31 semanas e 6 dias (22 casos, 53,7\%) de IG.

Tabela 2. Distribuição por faixas de peso ao nascer no grupo de pacientes com HPIV.

\begin{tabular}{ll}
\hline & HPIV (n) $(\%)$ \\
\hline$<500 \mathrm{~g}$ & 0 \\
$500-999 \mathrm{~g}$ & $15(36,6 \%)$ \\
$1000-1499 \mathrm{~g}$ & $20(48,8 \%)$ \\
$>=1500 \mathrm{~g}$ & $6(14,6 \%)$ \\
\hline
\end{tabular}

Tabela 3. Distribuição por IG no grupo de pacientes com HPIV.

\begin{tabular}{ll}
\hline & HPIV $(\mathrm{n})(\%)$ \\
\hline$<28 \mathrm{~s}$ & $12(29,3 \%)$ \\
$28-31 \mathrm{~s} 6 \mathrm{~d}$ & $22(53,7 \%)$ \\
$32-34 \mathrm{~s}$ & $7(17 \%)$ \\
\hline
\end{tabular}

Em relação ao uso antenatal de corticoide e de sulfato de magnesio, dos 110 pacientes incluídos no estudo (grupo com e sem HPIV), 85 (77,3\%) fizeram corticoide e 46 (41,8\%) fizeram sulfato de magnésio. Entre os 41 pacientes com HPIV, $31(75,6 \%)$ receberam corticoide (pelo menos 1 dose de dexametasona) e $14(34,1 \%)$ receberam sulfato de magnésio. Destes, em 11 casos $(78,6 \%)$, o uso dessa medicação associou-se à terapêutica de pré-eclâmpsia grave e eclâmpsia e, em outros 3 casos $(21,4 \%)$, não. Dos 41 que tiveram HPIV, $13(31,7 \%)$ receberam corticoide e sulfato de magnesio conjuntamente, $18(43,9 \%)$ receberam apenas corticoide, 1 $(2,4 \%)$ recebeu apenas sulfato de magnésio e 9 (21,9\%) não receberam nenhuma dessas medicações (Tabela 4). Os 2 casos de HPIV grau IV (4,9\%), receberam apenas corticoide e não receberam sulfato de magnésio.

Tabela 4. Uso antenatal de sulfato de magnésio e corticoide entre os pacientes com HPIV.

\begin{tabular}{|c|c|c|c|c|}
\hline \multirow[t]{3}{*}{$\mathbf{N}=41$} & \multicolumn{2}{|c|}{ S.Magnésio S.Magnésio } & \multicolumn{2}{|c|}{ S.Magnésio S.Magnésio } \\
\hline & SIM & NÃO & SIM & NÃO \\
\hline & $\begin{array}{l}\text { Corticoide } \\
\text { SIM }\end{array}$ & $\begin{array}{l}\text { Corticoide } \\
\text { SIM }\end{array}$ & $\begin{array}{l}\text { Corticoide } \\
\text { NÃO }\end{array}$ & $\begin{array}{l}\text { Corticoide } \\
\text { NÃO }\end{array}$ \\
\hline HPIV I (n=26) & $9(34,6 \%)$ & $10(38,5 \%)$ & $1(3,8 \%)$ & $6(23 \%)$ \\
\hline HPIV II $(n=12)$ & $3(25 \%)$ & $6(50 \%)$ & 0 & $3(25 \%)$ \\
\hline HPIV III (n=1) & $1(100 \%)$ & 0 & 0 & 0 \\
\hline HPIV IV (n=2) & 0 & $2(100 \%)$ & 0 & 0 \\
\hline $\begin{array}{l}\text { Total de HPIV } \\
(n=41)\end{array}$ & $13(31,7 \%)$ & $18(43,9 \%)$ & $1(2,4 \%)$ & $9(21,9 \%)$ \\
\hline
\end{tabular}

Acerca do tipo de parto, dos 41 casos de HPIV, 31 (75,6\%) nasceram através de parto cesárea e $10(24,4 \%)$ de parto vaginal. Houve predomínio do sexo masculino (22 casos, $53,7 \%$ ), em relação ao sexo feminino (19 casos, 46,3\%).

\section{DISCUSSÃO}

Investigar a prevalência de hemorragia peri-intraventricular entre os RN prematuros de um serviço de referência é de suma importância, pois reflete a qualidade da assistência fornecida a esses pacientes pela equipe multiprofissional (principalmente na $1^{\mathrm{a}}$ semana de vida, mais especificamente, nas primeiras $72 \mathrm{~h}$ de vida) e as intervenções às quais eles são submetidos. ${ }^{5}$

O recém-nascido prematuro é colocado, logo após o nascimento, em um ambiente potencialmente estressante e sujeito a inúmeras manipulações e procedimentos. Este cenário bastante adverso leva ao surgimento de estímulos dolorosos e danosos, interferindo na homeostasia de seu organismo e em seu neurodesenvolvimento. ${ }^{8,9} \mathrm{O}$ período de internamento desses pacientes, em muitos casos, prolongado, coincide com o processo peculiar e essencial de desenvolvimento, crescimento e maturação cerebral. ${ }^{5}$ 
Em geral, em unidades neonatais, é recomendada a realização de US transfontanelar em todos os recém-nascidos com menos de $1500 \mathrm{~g}$ de peso ao nascer e/ou com idade gestacional igual ou inferior a 34 semanas, sendo realizado em 2 ou 3 dias de vida e repetido de acordo com o achado diagnóstico. ${ }^{10}$

Vários fatores de risco estão relacionados com a gênese da HPIV, entre eles estão os maternos ou obstétricos, perinatais e próprios do RN. Os fatores de risco maternos estão envolvidos com as condições que levam ao parto prematuro, entre elas: assistência pré-natal inadequada, diabetes mellitus e gestação gemelar. Entre os fatores perinatais, podemos citar: parto vaginal e sofrimento fetal. Já entre os fatores próprios do RN, pode-se enumerar: prematuridade, menor peso ao nascimento, necessidade de manobras de reanimação ao nascer, síndrome do desconforto respiratório, situações que levem a variações de pressão arterial, ventilação mecânica, persistência do canal arterial, sepse, entre outros. ${ }^{11}$

Utilizado como tocolítico, na prevenção e no tratamento de convulsões na pré-eclâmpsia grave ou eclâmpsia, o sulfato de magnésio apresenta propriedades anti-inflamatórias, vasculares e neuroprotetoras. ${ }^{12}$ Ainda não foi completamente esclarecido o mecanismo exato pelo qual ele previne danos ao cérebro fetal, porém, há várias ações biologicamente plausíveis que podem contribuir para esse efeito. ${ }^{13}$

Em humanos, a droga é essencial em processos-chave intracelulares, tais como glicólise, fosforilação oxidativa, síntese proteica, agregação dos ácidos nucleicos e manutenção da integridade da membrana plasmática. Em animais, a ação do sulfato de magnésio ocorre por meio da prevenção do acometimento cerebral pós-hipóxico, ao bloquear a liberação excessiva de glutamato nos canais de cálcio e, além disso, previne a morte neuronal, diminuindo a atividade proinflamatória. Como o cérebro do neonato parece ser mais suscetível às lesões decorrentes da liberação do glutamato, as drogas que bloqueiam os seus receptores, como o sulfato de magnésio, podem reduzir o risco de injúria cerebral no período perinatal. ${ }^{12,13,14}$

Estudos randomizados evidenciaram que o corticoide antenatal associa-se à redução da morbimortalidade em prematuros, à diminuição dos casos de síndrome do desconforto respiratório, bem como está relacionado à melhor estabilidade hemodinâmica do $\mathrm{RN}$ e, consequentemente, ao efeito protetor contra a hemorragia peri-intraventricular. $\mathrm{O}$ mecanismo protetor exato não está completamente compreendido. Essa medicação além de atuar na maturação pulmonar, também fornece maior resistência à parede dos vasos da matriz germinativa, reduzindo o risco de sangramento, e, também, torna o fluxo sanguíneo cerebral mais estável. Por conseguinte, indica-se seu uso para todas as gestantes com IG menor que 34 semanas em risco de trabalho de parto prematuro. ${ }^{15}$

Em nosso estudo, observamos que a incidência de HPIV foi maior no grupo de RN com peso de nascimento entre 1000 - 1499g (48,8\%). A menor incidência de HPIV no grupo de extremo baixo peso $(<1000 \mathrm{~g})$ poderia ser justificada pelo maior número de pacientes com peso maior que $1000 \mathrm{~g}$ incluídos em nossa amostra total, superestimando esse resultado. $\mathrm{Na}$ literatura revisada, há estreita relação entre peso baixo ao nascimento e incidência de HPIV. ${ }^{16,17}$ Abreu e colaboradores $(2007)^{6}$ observaram um peso médio menor dos RN no grupo com HPIV em comparação ao grupo sem HPIV.

A idade gestacional de 28 - 31 semanas e 6 dias foi mais prevalente nos pacientes com HPIV $(53,7 \%)$. A idade gestacional deveria ser a variável mais relevante, quando comparada ao peso de nascimento, pois, entre 26 e 32 semanas, a vasculatura da matriz germinativa, local inicialmente envolvido na gênese da HPIV, é mais abundante. Após esse período, o risco de sangramento tende a diminuir pois a região em questão começará a regredir de forma progressiva. Contudo, a avaliação da idade gestacional baseia-se em métodos, muitas vezes, subjetivos: data da última menstruação, ultrassonografia obstétrica precoce e exame físico do recém-nascido. Portanto, devido a esse motivo, a variável mais comumente utilizada nos estudos que definem grupo de risco para HPIV é o peso do RN. ${ }^{5}$

No presente estudo, no grupo com HPIV, houve predomínio do sexo masculino $(53,7 \%)$, quando comparado ao feminino, resultado condizente com algumas publicações. ${ }^{18,19}$ Outros trabalhos não relataram diferença da incidência de HPIV associada ao sexo. ${ }^{20}$

Visualizamos, ainda, maior prevalência de HPIV em RN nascidos de parto cesárea $(75,6 \%)$ em relação ao vaginal em nosso estudo. Ment e colaboradores ${ }^{20}$ relataram uma significativa proteção do parto cesárea na ocorrência de HPIV, quando comparado ao vaginal, o que coincide com outros estudos. ${ }^{21}$ Neves et al ${ }^{22}$ observaram preponderância dos partos cesarianas em relação aos normais nos pacientes com menos de 2500 gramas com HPIV, porém tal resultado poderia ser explicado por uma possível apreensão por parte da equipe obstétrica em evitar a exposição do prematuro ao parto vaginal, conhecidamente associado à maior propensão para HPIV, e, além disso, também reflete maior tendência à realização de cesarianas nos anos 90 , período coincidente com o estudado.

Em relação ao uso antenatal de corticoide, podemos observar que uma porção significativa $(77,3 \%)$ da amostra incluída recebeu a medicação (pelo menos 1 dose). Tal dado poderia ser justificado pela adesão da equipe médica à recomendação primordial de realizar corticoide em todas as gestantes com IG menor de 34 semanas em risco de trabalho de parto, pois, além de acelerar a maturidade pulmonar, fornece maior estabilidade hemodinâmica ao prematuro e evita HPIV. Entretanto, encontramos taxa de exposição ao corticoide semelhante no grupo com HPIV $(75,6 \%)$. Esse fato poderia ser explicado pela maior inclusão de recém-nascidos expostos ao corticoide em nossa amostra total (desse modo, superestimando o resultado).

Em relação ao sulfato de magnésio antenatal, entre os 110 pacientes incluídos no estudo (grupo com e sem HPIV), menos da metade recebeu a referida medicação $(41,8 \%)$ antenatal, e, entre os 41 que tiveram HPIV, em 34,1\% deles 
o sulfato de magnésio foi administrado. Houve ainda uma grande associação do uso da referida medicação no tratamento de pré-eclâmpsia grave ou eclâmpsia (78,6\% dos pacientes). Portanto, no serviço estudado, verificou-se baixa adesão ao uso da medicação como neuroprotetor fetal. Esse fato pode ser atribuído à dificuldade no manejo da referida droga, já que esta possui efeitos colaterais indesejados amplamente conhecidos e pode levar a complicações, o que exige monitoramento clínico mais rigoroso do paciente.

Outra possível justificativa para a baixa adesão ao sulfato de magnésio seria a ausência de consenso sobre os regimes terapêuticos (doses, frequência e duração) e sobre a segurança de sua utilização como neuroprotetor fetal. ${ }^{13}$ Em estudo realizado por Canterino e colaboradores ${ }^{23}$ com amostra bem maior de pacientes que a nossa, verificou-se que, em crianças com peso ao nascer entre $500 \mathrm{~g}$ e $1750 \mathrm{~g}$, não houve diferença estatística significante quanto à incidência de HPIV entre o grupo exposto ao sulfato de magnésio e o não-exposto. Portanto, não se observou efeito protetor dessa medicação contra HPIV nestes pacientes. ${ }^{1}$

Uma metanálise publicada por Doyle et al. ${ }^{24}$ demonstrou notáveis resultados benéficos do sulfato de magnésio antenatal na diminuição de alterações motoras e de paralisia cerebral em

\section{REFERÊNCIAS}

1. Nitsche A, Kliemann R, Manfrim EB, Zeigelboin BS, Liberalesso P. Hemorragia cerebral em recém-nascidos de baixo peso e o uso de sulfato de magnésio pré-natal. Pediatr Mod. 2014;50(2):65-8.

2. Ballabh P. Intraventricular Hemorrhage in premature infants: mechanism of disease. Pediatr Res. 2010;67(1):1-8.

3. Ballabh P. Pathogenesis and prevention of intraventricular hemorrhage. Clin Perinatol. 2014;41(1):47-67.

4. Silveira RC, Procianoy RS. Lesões isquêmicas cerebrais no recém nascido pré-termo de muito baixo peso. J Pediatr. 2005;81(1 Supl.1):S23-32.

5. Marba ST, Caldas JP, Vinagre LE, Pessoto MA. Incidence of periventricular/intraventricular hemorrhage in very low birth weight infants: a 15-year cohort study. J Pediatr. 2011;87(6):505-11.

6. Abreu LC, Souza AM, Oliveira AG, Selestrin CC, Guerra MS, Ferreira $\mathrm{C}$, et al. Incidência de hemorragia peri-intraventricular em recém-nascidos pré-termo e a relação com o peso ao nascer. Rev Bras Crescimento Desenvolv Hum. 2007;17(2):24-30.

7. Papile LA, Burstein J, Burstein R, Koffler H. Incidence and evolution of subependymal and intraventricular hemorrhage: a study of infants with birth weights less than 1.500 gm. J Pediatr. 1978;92(4):529-34.

8. Als H, Duffy FH, McAnulty GB. Effectiveness of individualized neurodevelopmental care in the newborn intensive care unit (NICU). Acta Paediatr Suppl. 1996;416:21-30.

9. Vandenberg KA. Individualized neurodevelopmental care for high risk newborns in the NICU: a practice guideline. Early Hum Dev. 2007;83(7):433-42. neonatos prematuros expostos a essa medicação. No entanto, não foi observada modificação na taxa de mortalidade.

Futuros ensaios clínicos são necessários para maior esclarecimento a respeito do efeito protetor conferido pelo corticoide e pelo sulfato de magnésio antenatais na diminuição de HPIV, incluindo resultados no sistema nervoso central a longo prazo. Em relação ao corticoide antenatal, seu uso adequado e bem indicado em prematuros deve ser mais estimulado em razão dos seus amplos e apreciáveis benefícios. Estes estão relacionados à maior estabilidade desses pacientes tanto do ponto de vista respiratório,quanto do hemodinâmico, propiciando redução da mortalidade neonatal, do tempo de internamento hospitalar e da morbidade neurológica.

\section{CONCLUSÃO}

Podemos concluir que, em nossa instituição, a relação entre HPIV e prematuros com IG menor que 34 semanas corresponde a $37,3 \%$. A grande maioria dos casos de HPIV encontrados foram em $\mathrm{RN}$ com peso de nascimento menor que $1500 \mathrm{~g}(85,4 \%)$. A taxa de exposição ao corticoide na amostra total incluída foi de $77,3 \%$, semelhante à exposição no grupo com HPIV $(75,6 \%)$ e a exposição ao sulfato de magnésio foi de $41,8 \%$ na amostra total e $34,1 \%$ no grupo com HPIV.

10. Perlman JM, Rollins N. Surveillance protocol for the detection of intracranial abnormalities in premature neonates. Arch Pediatr Adolesc Med. 2000;154(8):822-6.

11. Brasil. Ministério da Saúde. Atenção à saúde do recém-nascido: guia para profissionais de saúde [internet]. Brasília: Ministério da Saúde; 2011; [acesso em: 2016 abr 25].

Disponível em: http://bvsms.saude.gov.br/bvs/publicacoes/atencao_ recem_nascido_\%20guia_profissionais_saude_v3.pdf

12. Santos AM, Meneguel JF, Guinsburg R. Hemorragia periintraventricular em recém-nascidos pré-termo extremo: uma complicação prevenível? In: Procianoy RS, Leone CR. Programa de atualização em neonatologia - PRORN. 2011;9(1):67-122.

13. Coutinho T, Coutinho CM, Coutinho LM. Sulfato de magnésio: um avanço na neuroproteção fetal? Femina. 2013;41(3):155-62.

14. The antenatal magnesium sulphate for neuroprotection guideline development panel. Antenatal magnesium sulphate prior to preterm birth for neuroprotection of the fetus, infant and child: national clinical practice guidelines [Internet]. Adelaide: The University of Adelaide; 2010 [acesso em: 2013 Ago 5]. Disponível em: http:// www.adelaide.edu.au/arch/MagnesiumSulphate2010.pdf

15. Vinagre LE, Marba ST. Uso antenatal do corticosteroide e hemorragia peri-intraventricular. Rev Paul Pediatr. 2010;28(3):346-52.

16. Ferriero DM. Neonatal brain injury. $N$ Engl $J$ Med. 2004;351(19):1985-95.

17. Farage L, Assis MC. Achados ultra-sonográficos da hemorragia intracraniana em recém-nascidos prematuros. Arq Neuropsiquiatr. 2005;63(3b):814-16. 
18. Tavares EC, Corrêa FF, Viana MB. Fatores de risco para hemorragias peri-intraventriculares em recém-nascidos com peso menor de 2000 gramas. J Pediatr. 1998;74(1):17-24.

19. Setzer ES, Webb IB, Wassenaar JW, Reeder JD, Mehta PS, Eitzman DV. Platelet dysfunction and coagulopathy in intraventricular hemorrhage in the premature infant. J Pediatr. 1982;100(4):599-605.

20. Ment LR, Oh W, Philip AG, Ehrenkranz, RA, Duncan CC, Allan $\mathrm{W}$, et al. Risk factors for early intraventricular hemorrhage in low birth weight infants. J Pediatr. 1992;121(5 Pt 1):776-83.

21. Leviton A, Fenton T, Kuban KC, Pagano M. Labor and delivery characteristics and the risk of germinal matrix hemorrhage in low birth weight infants. J Child Neurol. 1991;6(1):35-40.
22. Neves LA, Neto JA, Kneipp D, Fonseca LG, Rosado MP, Barreto MR. Hemorragia intracraniana no recém-nascido pré-termo. Casuística da UTI Neonatal do Hospital Albert Sabin. HU rev. 2007;33(2):47-52.

23. Canterino JC, Verma UL, Visitainer PF, Figueroa R, Klein SA, Tejani NA. Maternal magnesium sulfate and the development of neonatal periventricular leucomalacia and intraventricular hemorrhage. Obstet Gynecol. 1999;93(3):396-402.

24. Doyle LW, Crowther CA, Middleton P, Marret S, Rouse D. Magnesium sulphate for women at risk of preterm birth for neuroprotection of the fetus [Internet]. Cochrane Database of Systematic; 2009 [acesso em: 2016 abr 29]. Disponível em: http://onlinelibrary. wiley.com/doi/10.1002/14651858.CD004661.pub3/abstract

\section{Como citar:}

Vasconcelos SA, Lavor MF, Ferreira DM. Prevalência de hemorragia peri-intraventricular em recém-nascidos de muito baixo peso e/ou com idade gestacional menor que 34 semanas e relação com o uso antenatal de corticoide e de sulfato de magnesio em maternidade terciária. Rev Med UFC. 2017 mai-ago;57(2):14-19. 